\title{
Dynamically taming focal fields of femtosecond lasers for fabricating microstructures
}

\author{
Meng-Qiang Cai (蔡孟强) $)^{1,2}$ ， Qiang Wang (王 强) ${ }^{1}$, Cheng-Hou Tu (涂成厚 $)^{1,5 *}$, Yong-Nan Li (李勇男) $)^{1}$, and \\ Hui-Tian Wang (王慧田) $)^{3,4}$ \\ ${ }^{1}$ School of Physics and Key Laboratory of Weak Light Nonlinear Photonics, Nankai University, Tianjin 300071, China \\ ${ }^{2}$ Institute of Space Science and Technology, Nanchang University, Nanchang 330031, China \\ ${ }^{3}$ National Laboratory of Solid State Microstructures, Nanjing University, Nanjing 210093, China \\ ${ }^{4}$ Collaborative Innovation Center of Advanced Microstructures, Nanjing University, Nanjing 210093, China \\ ${ }^{5}$ Collaborative Innovation Center of Light Manipulations and Applications, Shandong Normal University, Jinan 250358, China \\ *Corresponding author: tuchenghou@nankai.edu.cn \\ Received May 28, 2021 | Accepted August 2, 2021 | Posted Online October 9, 2021
}

Micromachining based on femtosecond lasers usually requires accurate control of the sample movement, which may be very complex and costly. Therefore, the exploration of micromachining without sample movement is valuable. Herein, we have illustrated the manipulation of optical fields by controlling the polarization or phase to vary periodically and then realized certain focal traces by real-time loading of the computer-generated holograms (CGHs) on the spatial light modulator. The focal trace is composed of many discrete focal spots, which are generated experimentally by using the real-time dynamically controlled CGHs. With the designed focal traces, various microstructures such as an ellipse, a Chinese character "Nan", and an irregular quadrilateral grid structure are fabricated in the z-cut $\mathrm{LiNbO}_{3}$ wafers, showing good qualities in terms of continuity and homogeneity. Our method proposes a movement free solution for micromachining samples and completely abandons the high precision stage and complex movement control, making microstructure fabrication more flexible, stable, and cheaper.

Keywords: manipulation of optical fields; computer-generated holograms; focal trace; microstructures. DOI: 10.3788/COL202220.010502

\section{Introduction}

Polarization and phase, as the intrinsic nature of light, play important roles in optical field manipulation and various applications. By controlling the polarization state or phase of optical fields, novel optical fields can be obtained, e.g., vector optical field $^{[1]}$ and optical vortex ${ }^{[2]}$. Due to the fascinating properties after tight focusing, they are widely used in optical trapping and manipulation ${ }^{[3,4]}$, super-resolution imaging ${ }^{[5]}$, microfabrication $^{[6-8]}$, and so on.

As a promising tool for the fabrication of optical devices and microstructures, femtosecond laser processing has been successfully employed to process various materials such as metals, semiconductors, and dielectrics ${ }^{[9-16]}$ due to its characteristics of simplicity, flexibility, and high precision. In order to fabricate microstructures at the millimeter scale, an enormous number of processing points are needed; therefore, processing efficiency must be improved. To improve the micromachining efficiency, microlens arrays ${ }^{[17,18]}$ and parallel processing using multi-beam interference ${ }^{[19,20]}$ have been used to fabricate multi-microholes and two/three-dimensional microstructures. However, these methods are not flexible and cannot be used for processing more complex structures due to the fixed optical field pattern. To overcome the limitation, a spatial light modulator (SLM) displaying a computer-generated hologram $(\mathrm{CGH})$ has been used to manipulate the polarization/phase of optical fields to achieve variable optical fields and improve the processing efficiency and flexibility ${ }^{[7,8,21-29]}$. Normally, micromachining also requires a high precision translating stage to accurately control the sample movement, which may be very complex and costly. Therefore, the exploration of micromachining without sample movement is something to look forward to.

In this Letter, we have illustrated the manipulation of optical fields by controlling the polarization or phase periodically varying across the wavefront and then realized certain focal traces by loading dynamical (time-varying) CGH on the SLM. Based on the generated focal traces, we have successfully fabricated microstructures such as an ellipse, an irregular quadrilateral grid structure, and a Chinese character. In terms of the elliptic 
focal trace, we anticipated that it can be used to cut material along the elliptic trace to fabricate elliptic micro-tubes for microfluidics and to transport particles along the elliptic trace in optical tweezer technology. The benefit of our method is that no motion of samples is needed when fabricating microstructures, and it is more flexible to fabricate an arbitrary micropattern. More importantly, based on our method, any arbitrary freestyle focal trace can be formed. Unlike some methods that use iterative algorithms to generate specific holograms for control focal spots, our method is fast, accurate, and efficient.

\section{Principle}

Firstly, we illustrate the realization of a two-spot focal intensity distribution by manipulating the polarization state of an optical field and then the formation of an elliptic focal trace, which is used to fabricate elliptic microstructures. We should note the fact that any freestyle curvilinear trace can be formed based on this method, and herein we just take the formation of an elliptic focal trace as the example. For this purpose, we construct an optical field with the periodic variation in polarization along a certain direction in the pupil plane, as shown in Figs. 1(a) and $1(\mathrm{~b})$, which can be written as
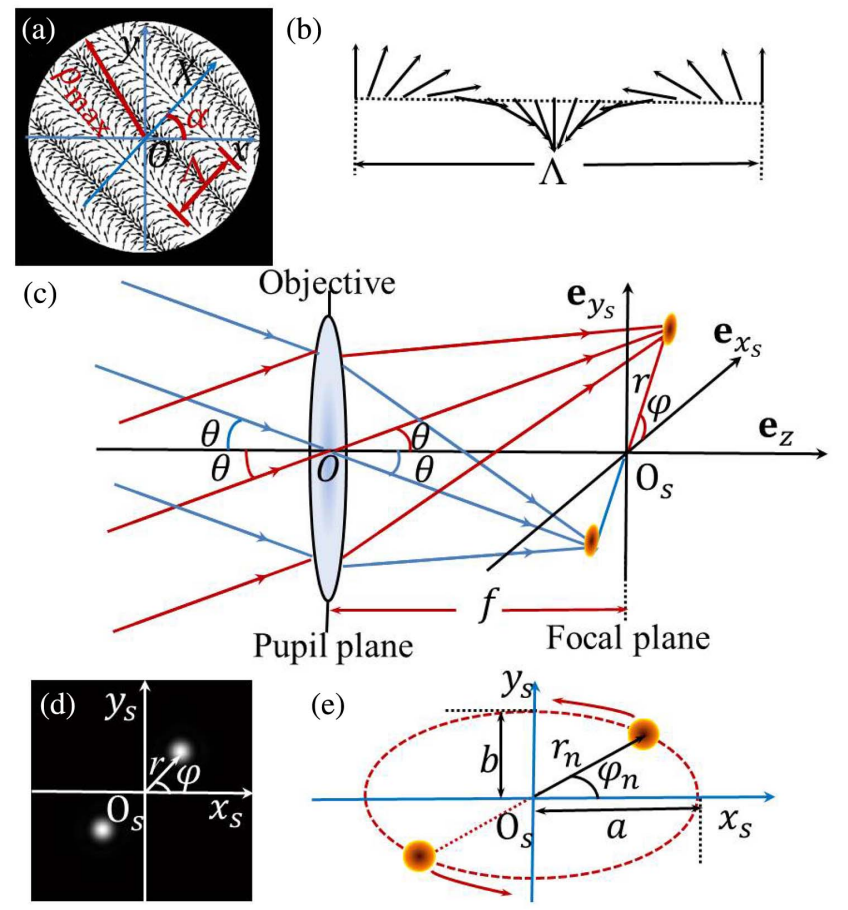

Fig. 1. Realization of a two-spot focal intensity distribution. (a) Schematic diagram of the polarization distribution for the constructed optical field, (b) the polarization variation within one period, (c) the focusing of the constructed optical field with periodic variation in polarization along certain directions in the pupil plane, (d) the corresponding intensity distribution of the focal field, and (e) the formation of an elliptic focal trace.

$$
\begin{aligned}
\mathbf{E} & =\cos (2 \pi X / \Lambda) \mathbf{e}_{x}+\sin (2 \pi X / \Lambda) \mathbf{e}_{y} \\
& =\frac{\sqrt{2}}{2}\left(e^{-i 2 \pi X / \Lambda} \mathbf{e}_{r}+e^{i 2 \pi X / \Lambda} \mathbf{e}_{l}\right)=\mathbf{E}_{1}+\mathbf{E}_{2},
\end{aligned}
$$

where $x$ and $y$ are the horizontal and vertical coordinates, respectively, and $\mathbf{e}_{x}$ and $\mathbf{e}_{y}$ are the unit vectors of the horizontal and vertical axes of the Cartesian coordinates in the pupil plane, respectively; $\Lambda$ is the period of the polarization variation, $X=$ $x \cos \alpha+y \sin \alpha$ represents the coordinate axis along the direction of polarization variation, and herein $\alpha$ is the included angle between the $X$ and $x$ axes, as shown in Figs. 1(a) and 1(b). $\mathbf{e}_{r}=$ $\frac{\sqrt{2}}{2}\left(\mathbf{e}_{x}+i \mathbf{e}_{y}\right)$ and $\mathbf{e}_{l}=\frac{\sqrt{2}}{2}\left(\mathbf{e}_{x}-i \mathbf{e}_{y}\right)$ are the right-handed and left-handed circularly polarized basis vectors, respectively. According to Eq. (1), we can see that the optical field $\mathbf{E}$ is formed by superposing the two circularly polarized flat-topped beams $\mathbf{E}_{1}$ and $\mathbf{E}_{2}$ in the pupil plane.

In Fig. 1(c), we can set the angle between the propagation direction of the beam $\mathbf{E}_{1}$ (or $\mathbf{E}_{2}$ ) and the optical axis ( $z$ axis) of the objective to be $\theta$ (or $-\theta$ ). As the wave vector is perpendicular to the equiphase surface in free space, we can get $\sin \theta=\lambda / \Lambda$ ( $\lambda$ is the wavelength). According to geometrical optics theory, the optical field $\mathbf{E}_{1}$ (or $\mathbf{E}_{2}$ ) can be focused into one spot, the distance $(r)$ between the spot and the coordinate origin $\left(O_{s}\right)$ in the focal plane is equal to $f \tan \theta$ (where $f$ is the focal length of the objective), and the azimuthal angle $\varphi$ of the spot in the coordinate system $x_{s} O_{s} y_{s}$ is equal to $\alpha$ (or $\left.\pi+\alpha\right)$ in the focal plane, as shown by Fig. 1(c), which can be written as $\varphi=\alpha$.

As the period $\Lambda$ is far greater than the wavelength $\lambda$, we can make an approximation $\sin \theta \approx \tan \theta$. Therefore, the relationship between the parameters $r$ and $\Lambda$ can be written as $\lambda / \Lambda=r / f$. Based on the Richard-Wolf diffraction integral theory ${ }^{[1]}$, the focal intensity distribution of the optical field $\mathbf{E}$, with the parameters $\Lambda=2 \rho_{\max } / 3$ (where $\rho_{\max }$ is the effective aperture radius), $\alpha=\pi / 4$, and the objective of $\mathrm{NA}=0.75$, is two centrosymmetric circular spots in the focal plane, as shown by Fig. 1(d). Moreover, the distance $(r)$ between the center of the focal spot and the origin $O_{s}$ is $\lambda f / \Lambda$. Therefore, we can control the position of focal spots in the focal plane through changing the parameters $\Lambda$ and $\alpha$ and make the focal spots move along the designed track, e.g., an ellipse.

To get an elliptic focal trace [see Fig. 1(e)], we just separate the whole ellipse into many discrete points for easy operation (in our case, we use 74 points) and hence simultaneously move the two focal spots point by point; as a result, the two focal spots just need to move 37 positions. The coordinates of a certain spot in the elliptic track can be written as $\left(a \cos t_{n}, b \sin t_{n}\right)$, where $a$, $b, t_{n}$, and $n$ are, respectively, the length of the half-major axis, the length of the half-minor axis, the angle to describe the point located at the elliptic trace, and the integer number of the point in Cartesian coordinates. Therefore, $t_{n}$ can be achieved through the formula of an elliptic arc length as

$$
n[\pi b+2(a-b)] / 37=\int_{0}^{t_{n}} \sqrt{a^{2} \cos ^{2} t+b^{2} \sin ^{2} t} \mathrm{~d} t .
$$


In the polar coordinates, the polar coordinates of the corresponding spot can be written as $\left(r_{n}, \varphi_{n}\right)$, and $\varphi_{n}$ and $r_{n}$ are as follows:

$$
\begin{gathered}
\varphi_{n}=\arccos \left(a \cos t_{n} / \sqrt{a^{2} \cos ^{2} t_{n}+b^{2} \sin ^{2} t_{n}}\right), \\
r_{n}=a b / \sqrt{b^{2} \cos ^{2} \varphi_{n}+a^{2} \sin ^{2} \varphi_{n}}
\end{gathered}
$$

where $n=1,2,3, \ldots, 37$. According to the equations $\lambda / \Lambda=r / f$ and $\varphi=\alpha$, the parameters $\Lambda$ and $\alpha$ of the corresponding incident optical field can be expressed as

$$
\begin{gathered}
\Lambda=\Lambda_{n}=\lambda f \sqrt{b^{2} \cos ^{2} \alpha_{n}+a^{2} \sin ^{2} \alpha_{n}} / a b, \\
\alpha=\alpha_{n}=\varphi_{n} .
\end{gathered}
$$

We set $a=2 b=8 \lambda$ and $f=\rho_{\max } / \mathrm{NA}$ (where NA $=0.75$ ) and substitute them into Eqs. (2)-(4) to get the values of $\Lambda$ and $\alpha$, which vary with $n$. As shown by Fig. 2, the arrows in Figs. 2(a)2(d) represent the polarization direction of the incident field, and the manipulation for different polarization states is shown from the left to right, which correspond to $n=1,13,25$, and 37, respectively, while Figs. 2(e)-2(h) show the focal planes of Figs. 2(a)-2(d) after focusing, respectively. It is obvious that the position of the focal point changes as $n$ increases. Figs. 2(i)-2(l) show the focal traces when $n$ are $1,13,25$, and 37, respectively. When the value of $n$ increases from 1 to 37, the two focal spots move along an ellipse, and finally the elliptic focal trace is formed, as shown in Fig. 2(1). Based on this method, any freestyle curvilinear trace can be formed, and here we just take the elliptic trace for example.

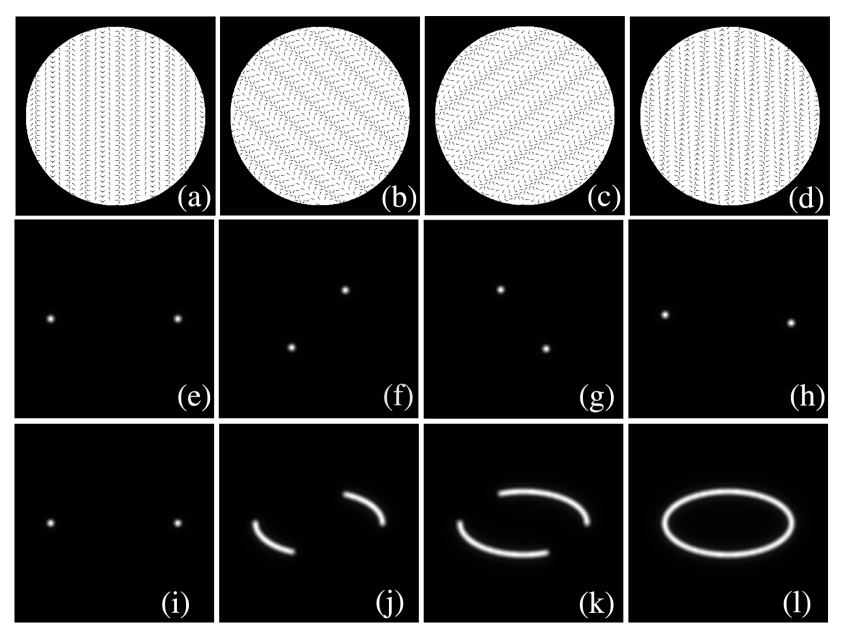

Fig. 2. Schematic diagrams of the elliptic focal trace formation. (a)-(d) are the polarization distributions and the periodic variation of the constructed optical field, and $n$ values are 1, 13, 25, and 37, respectively; (e)-(h) are the corresponding focal field distributions in the focal plane; (i)-(I) are the corresponding produced focal traces when $n$ are $1,13,25$, and 37, respectively. The size of (e)-(l) is $25 \times 25 \lambda^{2}$.

\section{Experimental Results and Discussion}

To fabricate a microstructure, as shown by Fig. 2(1), we firstly generate the optical field expressed in Eq. (1) in experiments and then dynamically vary the parameters of $\Lambda$ and $\alpha$ according to Eqs. (3) and (4) to generate the elliptical focal trace. The experimental setup is shown in Fig. 3(a) (within the dotted line), which is a common path interferometric configuration with the aid of a $4 f$ system composed of a pair of identical lenses (L1 and L2), based on the wavefront reconstruction ${ }^{[30]}$. An input linearly polarized light delivered from the femtosecond laser with the central wavelength of $800 \mathrm{~nm}$, pulse duration of $35 \mathrm{fs}$, and pulse repetition frequency of $1 \mathrm{kHz}$ (Coherent Inc.) is split into two parts, which are achieved by the \pm 1 st order beams diffracted from the CGH displayed on a phase-only SLM (P-SLM) (Holoeye PLUTO-NIR-2) with $1920 \times 1080$ pixels (each pixel has an $8 \mu \mathrm{m} \times 8 \mu \mathrm{m}$ size) and a frame rate of $60 \mathrm{~Hz}$, placed in the input plane of the $4 f$ system. The two parts carry the spatial variant phase, as shown in Figs. 3(b) and 3(c), respectively, and they pass through different optical paths, making them have orthogonal polarization states by using a pair of quarter wave plates behind the spatial filter (F1) placed in the Fourier plane of the $4 f$ system. The two orthogonally polarized parts are combined by a Ronchi phase grating $(G)$ placed in the output plane of the $4 f$ system. The transmission function of the CGH displayed on the SLM is shown in Fig. 3(d), which is a onedimensional grating function and can be written as

$$
t=\operatorname{circ}\left(\rho-\rho_{\max }\right)\left[0.5+0.5 \cos \left(2 \pi x / \Lambda_{0}+2 \pi X / \Lambda\right)\right]
$$

where $\Lambda_{0}$ is the Ronchi grating period, which is much less than $\Lambda$. $\operatorname{circ}\left(\rho-\rho_{\max }\right)$ is a circular function, which is equal to one as

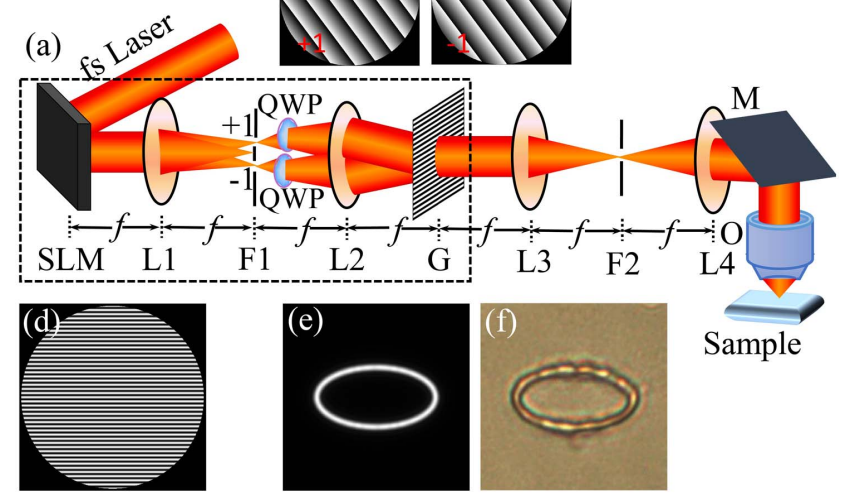

Fig. 3. Experiment setup and the corresponding details for the microstructure fabrication. (a) Schematic of experimental setup, (b) and (c) phase distributions for diffractive \pm 1 st-order beams, (d) transmission function of the CGH displayed on the SLM, (e) simulated elliptic focal trace, (f) microscopic imaging of the processed elliptic structure inside the $\mathrm{LiNbO}_{3}$ wafer through the transmitted illumination of white light. The sizes of $(e)$ and (f) are $20 \mu \mathrm{m} \times 20 \mu \mathrm{m}$. 
$\rho-\rho_{\max } \leq 0$ or 0 otherwise. The plane of the Ronchi grating and the pupil plane of objective $\mathrm{O}$ (Leica Inc., NA $=0.75$ ) are conjugate because of the second $4 f$ system composed of lenses L3 and L4 and a pinhole filter F2, so the optical fields in these two planes are equal.

$\mathrm{LiNbO}_{3}$, known as the "optical silicon" in the optoelectronic era, has been used as an ideal material for photoelectric chips/ integrated devices because of its excellent properties and comprehensive applications in optics and photonics, and hence it is the preferred sample material for our experiment. The optical field is focused beneath the surface of a $z$-cut $\mathrm{LiNbO}_{3}$ wafer about $20 \mu \mathrm{m}$. Just as mentioned above, when we vary $\Lambda$ and $\alpha$ according to Eq. (4), we can get 37 pairs of focal spots, which finally compose the elliptical focal trace. We know for every point of the focal trace, it corresponds to a pair of $\Lambda$ and $\alpha$ and hence a certain CGH, so we totally need 37 CGHs and then technically combine them to a video to be displayed on the SLM. For every CGH, the displayed time is about $0.02 \mathrm{~s}$, and the whole time spent is about $0.74 \mathrm{~s}$ to process an elliptical microstructure. When the energy density of the focal spot is above the threshold inside a $\mathrm{LiNbO}_{3}$ wafer, high-order nonlinear absorption allows the energy to be deposited predominantly, which consequently leads to the refractive index modification and induces the morphology change of processed materials ${ }^{[11]}$. In our experiment, we use a laser power a little higher than the threshold to fabricate the elliptical microstructure, and the experimental result is shown in Fig. 3(f). If comparing the experimental results with the simulation [Fig. 3(e)], we can see that the experimental result is quite good.

Secondly, we construct optical fields with linear and periodic variation in phase along a certain direction inspired from Eq. (1), which is as follows:

$$
\mathbf{E}=e^{i 2 \pi X / \Lambda} \mathbf{e}_{r}
$$

Based on the former analysis and description, we know the light field expressed by Eq. (6) can be focused into just one circular spot. Similarly, we can also change parameters of $\Lambda$ and $\alpha$ to control the position of the focal spot in the focal plane according to the equations $\lambda / \Lambda=r / f$ and $\varphi=\alpha$. With the aid of this fact, we can fabricate arbitrary structures by controlling the focal spot movement.

Supposing the microstructure to be fabricated occupies an area including $N_{1} \times N_{2}$ pixels (where $N_{1}$ and $N_{2}$ are the number of columns and rows, respectively), we then create a Cartesian coordinate system $x_{s} O_{s} y_{s}$ with the origin located at the center of the area, as shown in Figs. 4(a) and 4(b). The distance between two adjacent pixels is set to be $l_{0}$. Therefore, the coordinates of a given pixel, $\left(x_{n_{1}}, y_{n_{2}}\right)$, can be expressed as $\left(\left(n_{1}-N_{1} / 2\right) l_{0}\right.$, $\left.\left(N_{2} / 2-n_{2}\right) l_{0}\right)$, where the subscripts $n_{1}$ and $n_{2}$ represent the positional numbers of the column and row, respectively. Because a pixel has an area of $\left(l_{0}\right)^{2}$, herein we use the coordinates of the lower right vertex of a given pixel to define its coordinates. As a result, the polar coordinates $(r, \varphi)$ for the given pixel have the following expression:

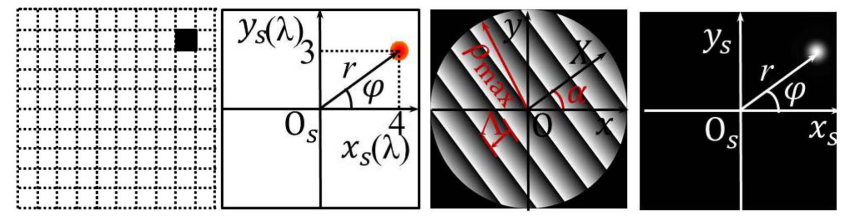

(a)

(b)

(c)

(d)

Fig. 4. Focal spot design by the phase manipulation. (a) A $10 \times 10$ pixels array with a pixel to be processed, (b) the needed focal spot in the focal plane, (c) the corresponding optical field with periodic variation in phase, and (d) the corresponding intensity distribution of the focal spot.

$$
\begin{aligned}
r=r_{n_{1}, n_{2}} & =\sqrt{\left(n_{1}-N_{1} / 2\right)^{2}+\left(N_{2} / 2-n_{2}\right)^{2}} l_{0}, \\
\varphi=\varphi_{n_{1}, n_{2}} & =\pi+\arctan \left(y_{n_{2}} / x_{n_{1}}\right), \\
& -\pi\left[\operatorname{sgn}\left(y_{n_{2}}\right)+\operatorname{sgn}\left(x_{n_{1}} y_{n_{2}}\right)\right] / 2 .
\end{aligned}
$$

According to the equations $\lambda / \Lambda=r / f$ and $\varphi=\alpha$, the parameters $\Lambda$ and $\alpha$ of the corresponding incident optical field can be expressed as

$$
\begin{gathered}
\Lambda=\Lambda_{n_{1}, n_{2}} \\
=\lambda f /\left(\sqrt{\left(n_{1}-N_{1} / 2\right)^{2}+\left(N_{2} / 2-n_{2}\right)^{2}} l_{0}\right), \\
\alpha=\alpha_{n_{1}, n_{2}}=\varphi_{n_{1}, n_{2}} .
\end{gathered}
$$

To fabricate the above microstructure, what we need to do is just use the coordinate of the pixel to be processed as that of the focal spot in the focal plane and then calculate the corresponding $\Lambda$ and $\alpha$ for the incident light field. Herein, we choose a microstructure with $10 \times 10$ pixels as the example. We choose a pixel whose coordinates are $\left(3 l_{0}, 4 l_{0}\right)$, as shown by Figs. $4(\mathrm{a})$ and $4(\mathrm{~b})$, while its polar coordinates are $\left(5 l_{0}, \arctan (0.75)\right)\left(l_{0}=\lambda\right)$ according to Eq. (7). Hence, the corresponding parameters $\Lambda$ and $\alpha$ for the incident light field are equal to $\rho_{\max } / 3.75$ and $\arctan (0.75)$, respectively, in the case of NA $=0.75$ according to Eq. (8). The phase variation of the incident optical field is shown in Fig. 4(c), and its focal field corresponds to Fig. 4(d).

Similar to the above example, herein we successfully fabricate a Chinese character and an irregular quadrilateral grid microstructure, which occupy $30 \times 30$ and $70 \times 70$ pixels, respectively. As shown in Figs. 5(a) and 5(d), the binary images (black) of the Chinese character "Nan" and irregular quadrilateral grid structure can be formed based on the pixel array. If each pixel in the array is treated as a single focal point, the combination patterns of these discrete focal points are shown in Figs. 5(b) and 5(e) correspondingly. In order to generate the microstructure pattern in experiment, we need to design the corresponding CGHs to generate the needed focal spots to process some certain pixels. Obviously, according to Eqs. (5), (7), and (8), we can get a series of time-varying CGHs to process all of the pixels. The designed time-varying CGHs are loaded on the SLM to generate phase manipulated optical fields based on the experimental 


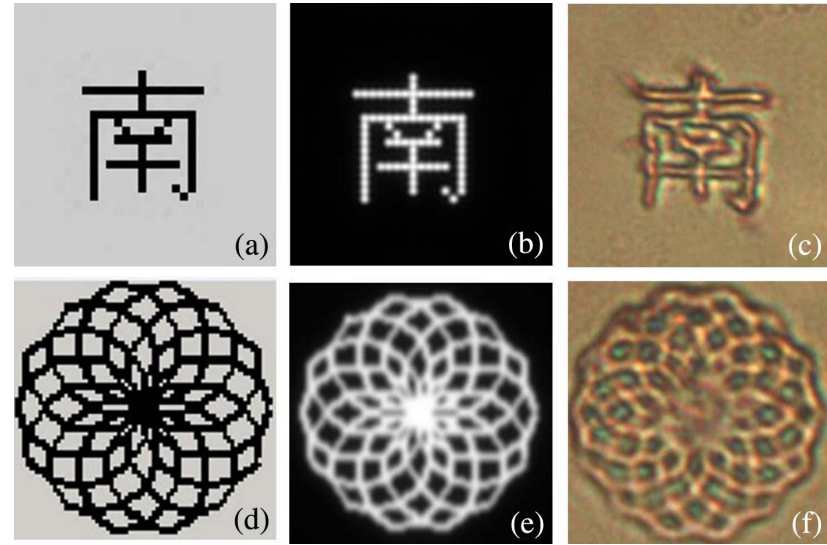

Fig. 5. Designed and fabricated microstructures. (a) and (d) are, respectively, the binary images with Chinese character "Nan" and irregular quadrilateral grid structure; (b) and (e) are the corresponding simulated patterns of the focal trace; (c) and (f) are the corresponding microscopy images of the fabricated structures. The sizes of (b), (c), (e), and (f) are $20 \mu \mathrm{m} \times 20 \mu \mathrm{m}$.

setup shown in Fig. 3(a). However, herein we must block the -1st-order beam to get the optical field expressed by Eq. (6). After focusing, the optical field is irradiated into a $\mathrm{LiNbO}_{3}$ wafer with the energy density slightly above the threshold. The fabricated Chinese character of "Nan" and irregular quadrilateral grid microstructure are shown in Figs. 5(c) and 5(f), respectively, and the pixel sizes for them are $l_{0}=0.77 \lambda$ and $l_{0}=0.34 \lambda$, respectively. The line width of the fabricated patterns is about $0.8 \mu \mathrm{m}$. Meanwhile, we can see that these microstructures are quite homogeneous and accorded with the simulation results.

\section{Summary}

In conclusion, we have designed and generated experimentally optical fields with periodical variation in polarization or phase of the wavefront and realized various focal traces, e.g., an ellipse, a Chinese character "Nan," and irregular quadrilateral grid structure patterns, which are achieved by loading dynamical CGHs on the SLM. Then, we use these focal traces, which vary dynamically, to interact with $\mathrm{LiNbO}_{3}$ wafers and get the corresponding microstructures with a line width about $0.8 \mu \mathrm{m}$. Our method provides a movement free dynamic programmable micromachining scheme, and any arbitrary freestyle focal trace can be generated and used to process microstructures, which is stable, cheap, and flexible. Moreover, it is also very promising in many applications, e.g., optical micro devices, optofluidic biochips, and photonic crystals.

\section{Acknowledgement}

This work was supported by the National Key R\&D Program of China (Nos. 2017YFA0303800 and 2017YFA0303700) and National Natural Science Foundation of China (Nos. 11534006, 11774183,12074196 , and 11904152).

\section{References}

1. Q. Zhan, "Cylindrical vector beams: from mathematical concepts to applications," Adv. Opt. Photon. 1, 1 (2009).

2. G. J. Gbur, Singular Optics (CRC Press, 2016).

3. Y. Zhang, J. Shen, C. Min, Y. Jin, Y. Jiang, J. Liu, S. Zhu, Y. Sheng, A. V. Zayats, and X. Yuan, "Nonlinearity-induced multiplexed optical trapping and manipulation with femtosecond vector beams," Nano Lett. 18, 5538 (2018).

4. X. Zhang, G. Rui, J. He, Y. Cui, and B. Gu, "Nonlinear accelerated orbiting motions of optical trapped particles through two-photon absorption," Opt. Lett. 46, 110 (2021).

5. Y. Kozawa, D. Matsunaga, and S. Sato, "Superresolution imaging via superoscillation focusing of a radially polarized beam," Optica 5, 86 (2018).

6. C. Hnatovsky, V. Shvedov, W. Krolikowski, and A. Rode, "Revealing local field structure of focused ultrashort pulses," Phys. Rev. Lett. 106, 123901 (2011).

7. K. Lou, S. X. Qian, Z. C. Ren, C. Tu, Y. Li, and H. T. Wang, "Femtosecond laser processing by using patterned vector optical fields," Sci. Rep. 3, 2281 (2013).

8. M. Cai, C. Tu, H. Zhang, S. Qian, K. Lou, Y. Li, and H.-T. Wang, "Subwavelength multiple focal spots produced by tight focusing the patterned vector optical fields," Opt. Express 21, 31469 (2013).

9. T. Jiang, S. Gao, Z. N. Tian, H. Z. Zhang, and L. G. Niu, "Fabrication of diamond ultra-fine structures by femtosecond laser," Chin. Opt. Lett. 18, 101402 (2020).

10. J. Dudutis, J. Pipiras, S. Schwarz, S. Rung, R. Hellmann, G. Račiukaitis, and P. Gečys, "Laser-fabricated axicons challenging the conventional optics in glass processing applications," Opt. Express 28, 5715 (2020).

11. K. M. Davis, K. Miura, N. Sugimoto, and K. Hirao, "Writing waveguides in glass with a femtosecond laser," Opt. Lett. 21, 1729 (1996).

12. B. W. Wu, C. Wang, Z. Luo, J. H. Li, S. Man, K. W. Ding, and J. A. Duan, "Controllable annulus micro-/nanostructures on copper fabricated by femtosecond laser with spatial doughnut distribution," Chin. Opt. Lett. 18, 013101 (2020).

13. A. Ancona, S. Döring, C. Jauregui, F. Röser, J. Limpert, S. Nolte, and A. Tünnermann, "Femtosecond and picosecond laser drilling of metals at high repetition rates and average powers," Opt. Lett. 34, 3304 (2009).

14. S. Xu, H. Fan, S.-J. Xu, Z.-Z. Li, Y. Lei, L. Wang, and J.-F. Song, "Highefficiency fabrication of geometric phase elements by femtosecond-laser direct writing," Nanomaterials 10, 1737 (2020).

15. M. K. Bhuyan, F. Courvoisier, P.-A. Lacourt, M. Jacquot, L. Furfaro, M. Withford, and J. Dudley, "High aspect ratio taper-free microchannel fabrication using femtosecond Bessel beams," Opt. Express 18, 566 (2010).

16. X. Li, M. Li, and H. J. Liu, "Effective strategy to achieve a metal surface with ultralow reflectivity by femtosecond laser fabrication," Chin. Opt. Lett. 19, 051401 (2021).

17. X. Q. Liu, L. Yu, S. N. Yang, Q.-D. Chen, L. Wang, S. Juodkazis, and H.-B. Sun, "Optical nanofabrication of concave microlens arrays," Laser Photon. Rev. 13, 1800272 (2019).

18. H. Fan, X.-W. Cao, L. Wang, Z.-Z. Li, Q.-D. Chen, S. Juodkazis, and H.-B. Sun, "Control of diameter and numerical aperture of microlens by a single ultra-short laser pulse," Opt. Lett. 44, 5149 (2019).

19. X. Jia, T. Jia, L. Ding, P. Xiong, L. Deng, Z. Sun, Z. Wang, J. Qiu, and $\mathrm{Z}$. Xu, "Complex periodic micro/nanostructures on $6 \mathrm{~h}$-sic crystal induced by the interference of three femtosecond laser beams," Opt. Lett. 34, 788 (2009).

20. T. Kondo, S. Matsuo, S. Juodkazis, V. Mizeikis, and H. Misawa, "Multiphoton fabrication of periodic structures by multibeam interference of femtosecond pulses," Appl. Phys. Lett. 82, 2758 (2003).

21. S. Hasegawa and Y. Hayasaki, "Polarization distribution control of parallel femtosecond pulses with spatial light modulators," Opt. Express 21, 12987 (2013).

22. Y. Jin, O. J. Allegre, W. Perrie, K. Abrams, J. Ouyang, E. Fearon, S. P. Edwardson, and G. Dearden, "Dynamic modulation of spatially structured polarization fields for real-time control of ultrafast laser-material interactions," Opt. Express 21, 25333 (2013). 
23. R. Drevinskas, J. Zhang, M. Beresna, M. Gecevičius, A. G. Kazanskii, Y. P. Svirko, and P. G. Kazansky, "Laser material processing with tightly focused cylindrical vector beams," Appl. Phys. Lett. 108, 221107 (2016).

24. M.-Q. Cai, P.-P. Li, D. Feng, Y. Pan, S.-X. Qian, Y. Li, C. Tu, and H.-T. Wang, "Microstructures fabricated by dynamically controlled femtosecond patterned vector optical fields," Opt. Lett. 41, 1474 (2016).

25. H. Lin, B. Jia, and M. Gu, "Dynamic generation of Debye diffraction-limited multifocal arrays for direct laser printing nanofabrication," Opt. Lett. 36, 406 (2011).

26. S. Hasegawa and Y. Hayasaki, "Holographic femtosecond laser processing with multiplexed phase Fresnel lenses displayed on a liquid crystal spatial light modulator," Opt. Rev. 14, 208 (2007).
27. Y. Hayasaki, T. Sugimoto, A. Takita, and N. Nishida, "Variable holographic femtosecond laser processing by use of a spatial light modulator," Appl. Phys. Lett. 87, 031101 (2005).

28. J. Ni, C. Wang, C. Zhang, Y. Hu, L. Yang, Z. Lao, B. Xu, J. Li, D. Wu, and J. Chu, "Three-dimensional chiral microstructures fabricated by structured optical vortices in isotropic material," Light Sci. Appl. 6, el7011 (2017).

29. B. Sun, P. S. Salter, C. Roider, A. Jesacher, J. Strauss, J. Heberle, M. Schmidt, and M. J. Booth, "Four-dimensional light shaping: manipulating ultrafast spatiotemporal foci in space and time," Light Sci. Appl. 7, 17117 (2018).

30. X.-L. Wang, J. Ding, W.-J. Ni, C.-S. Guo, and H.-T. Wang, "Generation of arbitrary vector beams with a spatial light modulator and a common path interferometric arrangement," Opt. Lett. 32, 3549 (2007). 